\title{
Multimode Storage of Quantum Microwave Fields in Electron Spins over 100 ms
}

\author{
V. Ranjan $\odot,{ }^{1}$ J. O’Sullivan $\odot,{ }^{2}$ E. Albertinale, ${ }^{1}$ B. Albanese,${ }^{1}$ T. Chanelière, ${ }^{3}$ T. Schenkel, ${ }^{4}$ \\ D. Vion $\odot,{ }^{1}$ D. Esteve $\odot,{ }^{1}$ E. Flurin, ${ }^{1}$ J. J. L. Morton, ${ }^{2}$ and P. Bertet ${ }^{1, *}$ \\ ${ }^{1}$ Université Paris-Saclay, CEA, CNRS, SPEC, 91191 Gif-sur-Yvette Cedex, France \\ ${ }^{2}$ London Centre for Nanotechnology, University College London, London WC1H OAH, United Kingdom \\ ${ }^{3}$ Université Grenoble Alpes, CNRS, Grenoble INP, Institut Néel, 38000 Grenoble, France \\ ${ }^{4}$ Accelerator Technology and Applied Physics Division, Lawrence Berkeley National Laboratory, \\ Berkeley, California 94720, USA
}

(Received 24 June 2020; accepted 19 October 2020; published 20 November 2020)

\begin{abstract}
We report long coherence times (up to $300 \mathrm{~ms}$ ) for near-surface bismuth donor electron spins in silicon coupled to a superconducting microresonator, biased at a clock transition. This enables us to demonstrate the partial absorption of a train of weak microwave fields in the spin ensemble, their storage for $100 \mathrm{~ms}$, and their retrieval, using a Hahn-echo-like protocol. Phase coherence and quantum statistics are preserved in the storage.
\end{abstract}

DOI: 10.1103/PhysRevLett.125.210505

Quantum memory as a matter-based information storage medium for itinerant qubits is a powerful ingredient in quantum technologies. In the optical domain [1-3], it underpins applications such as quantum repeaters. Quantum memories suitable for interfacing with superconducting quantum processors must operate in the microwave regime, which requires operation at millikelvin temperatures in a dilution refrigerator. Such devices could be used to operate a quantum Turing machine architecture with high internal connectivity and built-in long-term memory [Fig. 1(a) and Refs. [4,5]] helping to overcome some of the limitations of present-day superconducting quantum processors [6-8].

For implementing a microwave quantum memory, superconducting microwave cavities $[9,10]$ and mechanical resonators $[11,12]$ have been considered, with storage times in the millisecond range. Ensembles of electron spins in solids offer a large number of degrees of freedom well decoupled from their environment with coherence times that can reach seconds [13-15], and are thus well suited to implement a many-mode quantum memory [5,16-18]. For modularity, it is natural to physically separate the quantum processor and the spin-ensemble quantum memory, and to interface them via propagating microwave photons. Memory operation then consists in absorbing the incoming photons and releasing them on demand in the same quantum state [Fig. 1(a)].

A convenient way to interface the spins and the incoming photons [Fig. 1(b)] is via a superconducting microresonator [19-32] of frequency $\omega_{0}$, capacitively coupled to the input line with an energy damping rate $\kappa$, and inductively coupled (with single spin coupling strength $g_{0}$ ) to an ensemble of $N$ spins, characterized by its Larmor frequency $\omega_{S}$ with inhomogeneous linewidth $\Gamma$. The resonator serves to (a)

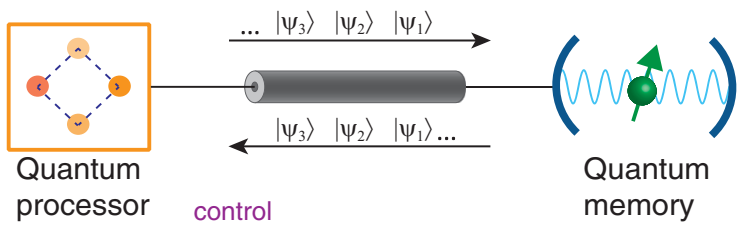

(b)

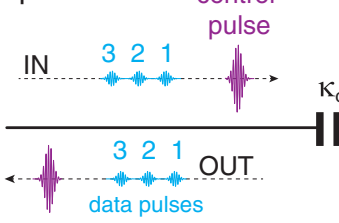

(c)

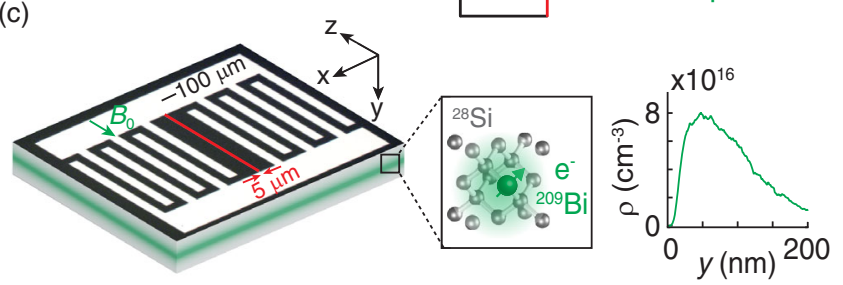

FIG. 1. Quantum memory. (a) The proposed architecture consisting of a quantum processor coupled via a coaxial cable to a quantum memory from electron spins. (b) Circuit implementation: an ensemble of $N$ electron spins are inductively coupled to a lumped resonator which is capacitively coupled to a microwave line. Weak coherent data pulses in our demonstration, travel along the lines, are stored by the spin ensemble, and then released on demand by a control pulse. The coupling strength of an individual spin to the resonator is $g_{0}$. (c) Device setup: The resonator is fabricated on top of the silicon substrate in a superconducting aluminum thin film to minimize internal losses. It consists of a capacitor shunted by a $5 \mu \mathrm{m}$-wide inductance wire, to which the spins of bismuth donors implanted around a depth $\sim 100 \mathrm{~nm}$ are inductively coupled. A magnetic field $B_{0}$ is applied along the inductor. 
enhance microwave absorption and reemission by spins, but also provides a convenient reset mechanism for the memory, via the Purcell relaxation of each individual spin at a rate $\Gamma_{P}=4 g_{0}^{2} / \kappa[33-35]$.

The physics of the memory can be demonstrated using weak resonant coherent pulses with a small average photon number. Such microwave pulses with amplitude $\beta_{\text {in }}(t)$ are absorbed by the spins with an efficiency governed by the ensemble cooperativity $C=N \Gamma_{P} / \Gamma$. Since the reflected amplitude is $\beta_{\text {ref }}(t)=\beta_{\text {in }}(t)(1-C) /(1+C)$, complete absorption is achieved for $C=1$, which appears as a necessary condition for a high-fidelity memory (see Refs. [36,37] and Supplemental Material [38]). The simplest retrieval sequence consists in applying a $\pi$ pulse to the spins after a delay $\tau$, which generates an echo of the absorbed pulse at time $2 \tau$. Because this echo is generated when nearly all spins are in the excited state, it is unavoidably accompanied by $N \Gamma_{P} / \Gamma=C$ spontaneously emitted noise photons, thus reducing the memory fidelity [39]. Therefore a more complex protocol must be used, involving two $\pi$ pulses and dynamic control of the cavity frequency, in order to form the echo in the spin ground state and thus avoid added noise [36,40].

Reaching unit cooperativity requires large spin concentrations, but spin-spin interactions then reduce the coherence time. This proved to be a serious limitation in previous experiments storing microwaves in spin ensembles [41-45], where the longest storage times demonstrated reached only of order $100 \mu \mathrm{s}$. This conflict can be mitigated by biasing the spins at special magnetic fields where their dc magnetic susceptibility vanishes, which minimizes spinspin interactions, whereas the ac susceptibility and thus also $g_{0}$ remain nonzero, opening the possibility to reach $C=1$ without compromising the coherence time. Such "clock transition" (CT) or "ZEro-First-Order-Zeeman" (ZEFOZ) points occur in spin systems where the electron spin is strongly hybridized with a nuclear spin by the hyperfine interaction, as in bismuth donors in silicon [46-49], rare-earth-ion-doped crystals [50,51], and molecular spins [52].

While all reports of CT coherence time enhancement so far were measured using bulk-doped samples [48,50-52], quantum information processing requires nanostructured devices, which may impact spin decoherence in a nontrivial way. Here, we demonstrate coherence time enhancement at a CT for implanted bismuth donors in silicon, coupled to a superconducting microresonator suitable for a microwave quantum memory, enabling us to demonstrate the long-term storage of microwave fields. Bismuth atoms were implanted around a $\sim 100 \mathrm{~nm}$ depth in a silicon substrate [Fig. 1(c)] that was enriched with the nuclear-spin-free ${ }^{28} \mathrm{Si}$ isotope for longer coherence time [53]. At low temperature, bismuth atoms trap a conduction electron, forming the donor systems. The spin Hamiltonian $H_{\mathrm{Bi}} / \hbar=$ $\left(\gamma_{e} \mathbf{S}+\gamma_{\mathrm{n}} \mathbf{I}\right) \cdot \mathbf{B}_{0}+A \mathbf{S} \cdot \mathbf{I}$ is the sum of the Zeeman interaction of the electron (nuclear) spin $S=1 / 2$ $(I=9 / 2)$ of the bismuth donor with the applied magnetic field $B_{0}\left(\gamma_{e} / 2 \pi \simeq 28 \mathrm{MHz} / \mathrm{mT}\right.$ and $\gamma_{n} / 2 \pi \simeq 7 \mathrm{kHz} / \mathrm{mT}$ being the electronic and nuclear gyromagnetic ratios) and of their hyperfine interaction with a strength $A / 2 \pi=1.475 \mathrm{GHz}$. The resulting energy levels $|F, m\rangle$ can be grouped in a low-energy $(F=4)$ manifold of 9 states and a high-energy $(F=5)$ manifold of 11 states [Fig. 2(a)], separated by $\sim 7.38 \mathrm{GHz}, m$ being the eigenvalue of the total angular momentum $S_{z}+I_{z}$ along the field direction $z[46,47]$. The operator $S_{x}$ has nonzero matrix elements between all pairs of states that verify $\Delta m= \pm 1$. We note that transitions $|4, m\rangle \leftrightarrow|5, m-1\rangle$ and $|4, m-1\rangle \leftrightarrow|5, m\rangle$ are quasidegenerate. In this work we are interested in the $|4,0\rangle \leftrightarrow|5,-1\rangle$ and $|4,-1\rangle \leftrightarrow|5,0\rangle$ transitions, which satisfy the CT condition at $\sim 7.338 \mathrm{GHz}$ and $B_{0}=27 \mathrm{mT}$ while the transition matrix elements $\left\langle 4,0\left|S_{x}\right| 5,-1\right\rangle=\left\langle 4,-1\left|S_{x}\right| 5,0\right\rangle=0.25$. To describe the interaction with microwave fields close to resonance, we model the pair of transitions as independent spin- $1 / 2$ systems labeled generically as $|0\rangle(|1\rangle)$ for the ground (excited) state.

The resonator is designed such that its resonance $\omega_{0}$ is close to the CT frequency of $7.338 \mathrm{GHz}$. Because of the kinetic inductance contribution to the resonator inductance, $\omega_{0} / 2 \pi$ decreases with $B_{0}$, reaching $7.336 \mathrm{GHz}$ at $27 \mathrm{mT}$. The total energy damping rate $\kappa=\kappa_{c}+\kappa_{i}$ includes the coupling rate $\kappa_{c}=4 \times 10^{5} \mathrm{~s}^{-1}$ and the power-dependent internal loss $\kappa_{i}$ [38], with $\kappa_{i} / \kappa \sim 0.7$ for $\sim 1$ intracavity photon and $\kappa_{i} / \kappa \sim 0.5$ at high power, due to two-level systems (TLS).

Spin spectroscopy is performed with a custom-built spectrometer described in more detail in Ref. [54], using the microresonator for the inductive spin detection, at $T=20 \mathrm{mK}$ so that $\hbar \omega_{0} \gg k T$. Hahn-echo sequences of pulses $(\pi / 2-\tau-\pi-\tau-e c h o)$ are sent to the resonator input at $\omega_{0}$. The resulting echo is amplified by a Josephson traveling wave parametric amplifier (JTWPA) at $20 \mathrm{mK}$ that adds noise close to the quantum limit [55], before further amplification at higher temperatures and demodulation at room temperature. Figure 2(c) shows the echo integral $A_{e}\left(B_{0}\right)$ around the CT. A spin signal is observed over a wide range of $B_{0}$ values around the CT, despite the detuning between $\omega_{0}$ and the expected donor frequency. This is explained by the differential thermal contractions of the resonator thin film with respect to the underlying substrate, which causes spatial variations of the strain profile and consequently of the donor hyperfine constant and the Larmor frequency [56,57]. Based on the measured lineshape for the first transition near $1.5 \mathrm{mT}$ [inset of Fig. 2(b)], the echo-signal field dependence of Fig. 2(c) is semiquantitatively accounted for [38].

The bismuth donor coherence time at the $\mathrm{CT}$ is obtained by measuring $A_{e}(\tau)$ at $B_{0}=27 \mathrm{mT}$ [Fig. 2(d)]. An exponential fit yields $T_{2}^{E}=0.30 \mathrm{~s}$, to our knowledge the 


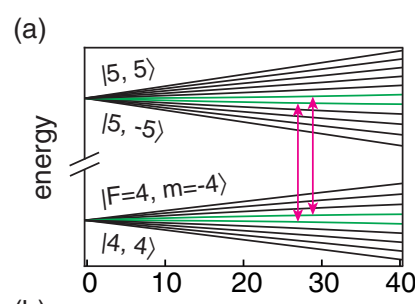

(b)
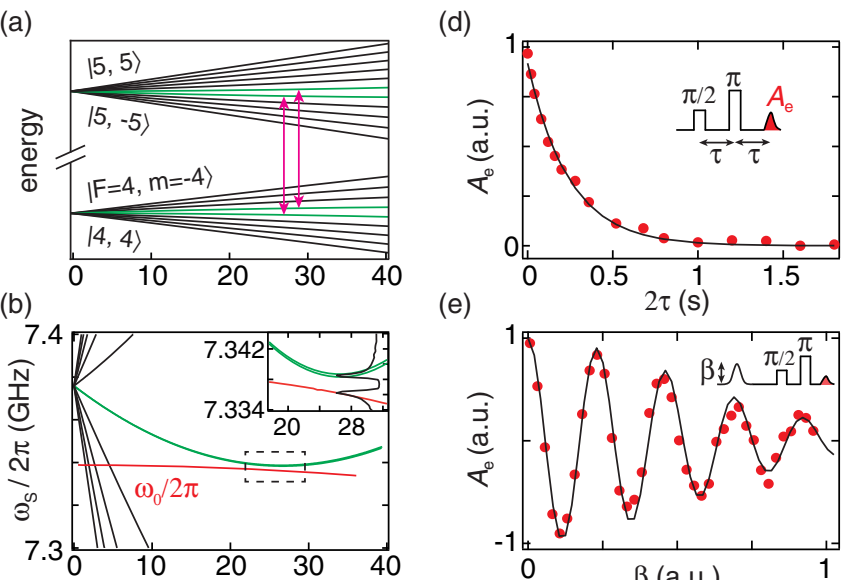

(c)

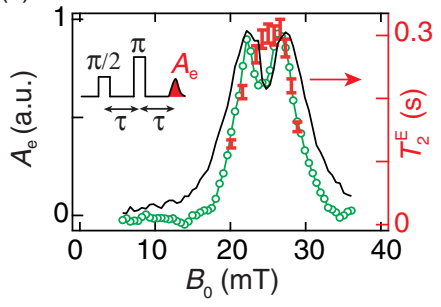

(f)
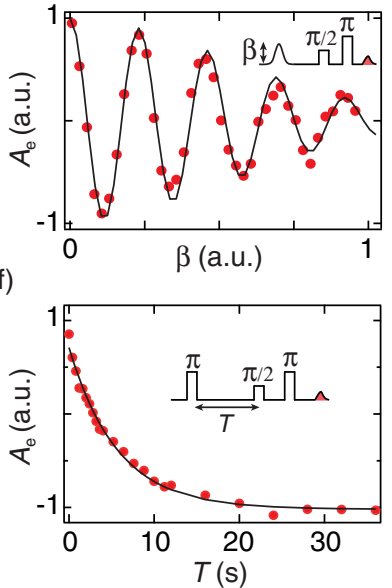

FIG. 2. Clock transition in bismuth and its characterization. (a) Energy level diagram $|F, m\rangle$ of electron spins from bismuth at small magnetic fields. Green lines indicate the four levels $|4,-1\rangle,|4,0\rangle,|5,-1\rangle$, and $|5,0\rangle$ involved in the two quasidegenerate CT. (b) The ESR-allowed transition frequency of bismuth donor spins in silicon between 7.3-7.4 GHz, and the measured resonator frequency $\omega_{0}$ as a function of $B_{0}$. The CT with $d \omega / d B_{0}=0$ can be seen at $B_{0}=27 \mathrm{mT}$. Inset: zoom around $\mathrm{CT}$ to show degenerate transition and expected strain broadened spectrum in frequency. (c) Measured echo detected spectroscopy around the CT (green symbols, left axis), calculated one (black line, left axis, see Ref. [38]), and two-pulse echo coherence time $T_{2}^{E}$ versus $B_{0}$ (red bars, right axis). (d) Measured echo area decay at CT (symbols) and corresponding exponential fit (line) yielding $T_{2}^{E}=0.3 \pm 0.05 \mathrm{~s}$. Magnitude detection is employed to circumvent phase noise from the measurement setup. (e) Measured (symbols) and simulated (line) Rabi oscillations at the CT. Each $A_{e}$ is plotted as a function of the amplitude $\beta$ of the first pulse. (f) Measured spin relaxation at the CT using an inversion recovery sequence (symbols), and corresponding exponential fit (line) yielding $T_{1}^{E}=5.5 \pm 0.5 \mathrm{~s}$.

longest coherence time measured for electron spins close to an interface. In comparison, the coherence time in a bulk bismuth-doped silicon sample at another CT was found to be $T_{2}^{E} \simeq 10^{3} \mathrm{~s} \cdot \mu \mathrm{m}^{-3} /[\mathrm{Bi}: \mathrm{Si}]$, with [Bi:Si] the donor concentration expressed in $\mu \mathrm{m}^{-3}$, due to direct flip flops between donors [48]. This process appears to be suppressed in our device where it would otherwise limit the coherence time to only $\sim 25 \mathrm{~ms}$ for our $[\mathrm{Bi}: \mathrm{Si}] \sim 4 \times 10^{4} \mu \mathrm{m}^{-3}$, likely due to locally inhomogeneous strain shifts caused by the microstructure [58]. If this broadening is smaller than the cavity linewidth (which seems to be approximately the case here), it has no impact on the cooperativity $C$, implying that long values of $T_{2}^{E}$ may indeed be compatible with a large cooperativity. Instead of direct flip flops, $T_{2}^{E}$ appears to be limited by charge noise at the interface between silicon and silicon oxide [59]. As expected, $T_{2}^{E}$ is longest near $B_{0}=27 \mathrm{mT}$, where $d \omega / d B_{0}=0$ [Fig. 2(c), right axis]; for comparison, we measure $T_{2}^{E}=7.5 \mathrm{~ms}$ on the $|4,-4\rangle \leftrightarrow$ $|5,-5\rangle$ transition at $B_{0}=1.4 \mathrm{mT}$ with $d \omega / d B_{0} \sim \gamma_{e}$. The Purcell spin relaxation time $T_{1}=5.5 \mathrm{~s}$ is measured using an inversion recovery sequence [Fig. 2(f)], yielding a spinphoton coupling constant $g_{0} / 2 \pi=40 \mathrm{~Hz}$. All the experiments described in the following are therefore averaged with a repetition time of $3 T_{1} \sim 16 \mathrm{~s}$.

We now demonstrate the coherent absorption and retrieval of weak microwave pulses by the donors at the CT. A Gaussian incoming pulse envelope $\beta_{\text {in }}(t)=$ $\beta \exp \left[-\left(t / t_{0}\right)^{2}\right]$ is chosen, with $t_{0}=10 \mu \mathrm{s}$ larger than the cavity field decay time $2 \kappa^{-1}=1.5 \mu \mathrm{s}$. To calibrate the input pulse intensity in photon number $n_{\text {in }}=$ $\int\left|\beta_{\text {in }}(t)\right|^{2} d t$, we compare the measured Rabi nutation of the donor spins as a function of $\beta$ [Fig. 2(e)], with numerical simulations in which all the parameters are determined experimentally. Next, we determine the ensemble cooperativity by measuring the ratio $\alpha^{p}(t) / \alpha^{s}(t)=$ $1 /(1+C)$ between the intracavity field $\alpha^{p}(t)\left[\alpha^{s}(t)\right]$ with spins polarized (unpolarized). The data shown in Fig. 3(a) yield $C=3.5 \pm 1 \times 10^{-2}$, corresponding to a spin density $N /(\Gamma / 2 \pi) \sim 10^{6}$ spins $/ \mathrm{MHz}$ consistent with the known sample implantation parameters. Note that a careful account of the contribution from resonator losses and TLS was necessary in the analysis [38].

With the knowledge of the cooperativity $C$, the whole storage and retrieval protocol can be demonstrated and quantitatively understood. Because $C \ll 1$, we use here the simple retrieval protocol based on a single $\pi$ pulse, with a square shape of $2 \mu$ s duration. For $n_{\text {in }}=240 \pm 24$ input photons, $\zeta n_{\text {in }}$ should be absorbed, with $\zeta=4 C\left(\kappa_{c} / \kappa\right)=$ $4.3 \pm 1.4 \times 10^{-2}$ [38], and $\zeta^{2} n_{\text {in }}=0.45 \pm 0.15$ photons should be reemitted as a Hahn echo. Data shown in Fig. 3(b) for $\tau=100 \mu$ s are in quantitative agreement with these analytical predictions as well as with a complete simulation of the experiment.

We then demonstrate long-lived and multimode first-in (last-out) microwave storage by sending a train of 20 weak Gaussian pulses with varying phases, and retrieving them using a single refocusing pulse. The experiment was performed twice, comparing input pulse intensities of $n_{\text {in }}=$ $240 \pm 24$ and $24 \pm 2$ photons. As seen in Fig. 3(c), echoes are retrieved after $100 \mathrm{~ms}$ with a well-defined phase. The retrieved field amplitude is slightly reduced from the expected value of $\zeta \beta_{\text {in }}$, mostly due to spin decoherence during the storage time, and also for a small part to resonator phase noise caused by vortices trapped in the resonator thin film [38]. The recovered intensity corresponds to, respectively, $0.3 \pm 0.1$ and $0.03 \pm 0.01$ photons per echo, $\sim 10^{-3}$ of the input pulse energy. 

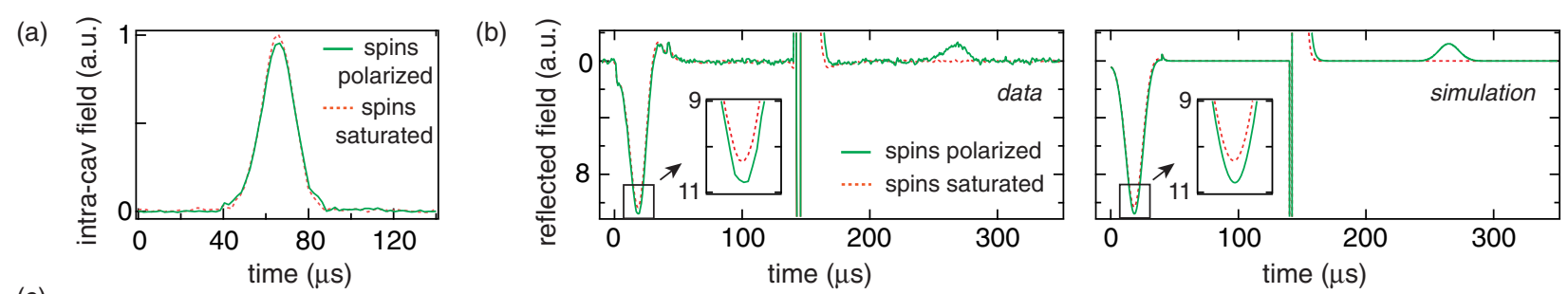

(c)

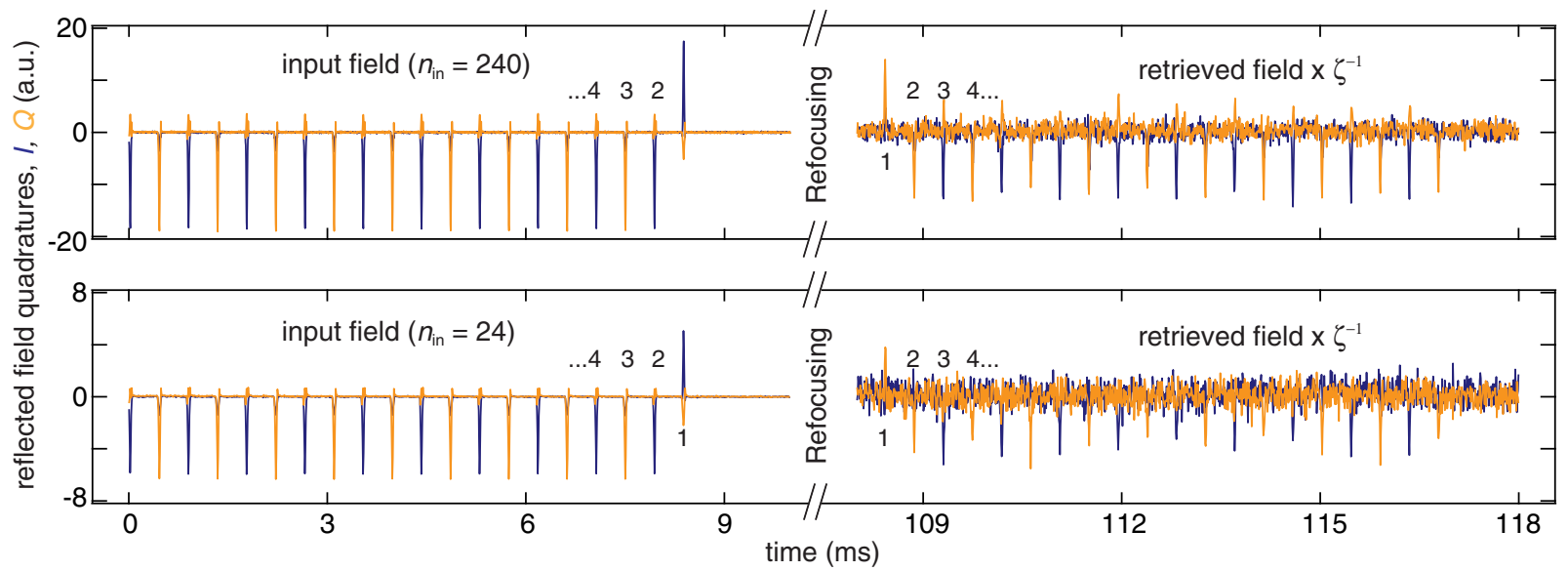

FIG. 3. Storage and retrieval of weak pulses at the clock transition $\left(B_{0}=27 \mathrm{mT}\right)$. (a) Measured intracavity field $\alpha$ averaged over $10^{3}$ repetitions at a repetition rate of $200 \mathrm{~ms}$ (spins saturated, dashed curve) and of $16 \mathrm{~s}$ (spins polarized, solid curve). (b) Measured (left panel, $4 \times 10^{3}$ averages) and simulated (right panel) reflected field on resonance $\left(\sqrt{\kappa_{c}} \alpha-\beta_{\text {in }}\right)$ and echo at a time $\ll T_{2}^{\mathrm{E}}$. The simulation assumes a spectral density of $N /(\Gamma / 2 \pi)=10^{6}$ spins $/ \mathrm{MHz}$ and a fixed $g_{0} / 2 \pi=40 \mathrm{~Hz}$. For panels (a), (b) there are 240 photons in the input pulse. (c) Top (bottom): a train of weak microwave fields, containing 240 (24) photons at the input and their retrieval after the refocusing $\pi$ pulse. The numbering highlights that retrieval maintains the phase relation with respect to the input field. The retrieved fields are multiplied by $\zeta^{-1} \approx 24$, where $\zeta=4 C\left(\kappa_{C} / \kappa\right)$ is the theoretical efficiency of the retrieved field at a time $\ll T_{2}^{E}$. Note that the input fields are measured at a frequency shifted by $5 \mathrm{MHz}$ from $\omega_{0} / 2 \pi$ to enable a direct comparison with the retrieved fields.

Finally, we address the question of the quantum statistics of the echo field. For that, we record a histogram of integrated output signals acquired during the echo $E$, and outside the echo $O$ at a time when all spins are in their ground state (Fig. 4). Thanks to the photon number calibration, the average echo amplitude $\bar{E}-\bar{O}=\zeta \sqrt{n_{\text {in }}}=0.5 \pm 0.1$ provides an absolute calibration of the horizontal scale in dimensionless (square root of photon number) units. This enables a comparison of the measured standard deviations $\sigma_{O} \sim \sigma_{E}=1.5 \pm 0.3$ to the expected $\sigma_{O}=\sqrt{\left(\bar{n}_{\mathrm{id}}+1\right) / 2}$ and $\sigma_{E}=$ $\sqrt{\left(\bar{n}_{\text {id }}+C+1\right) / 2}$ [38], where $\bar{n}_{\text {id }}$ describes JTWPA nonideality, microwave losses between the sample and the amplifier, and added noise by the higher-temperature amplification chain. We find $\bar{n}_{\text {id }}=3.5 \pm 1.7$, a reasonable value compared to those measured in similar circuit QED setups. Then, no measurable difference is observed between $\sigma_{E}$ and $\sigma_{O}$, in agreement with the theoretical estimate which predicts less than $0.5 \%$ difference. Overall, these measurements prove the consistency between our photon number calibration protocol and the statistics of the recovered signal, and they show that the echo is recovered with negligible added noise, close to the quantum limit. (a)

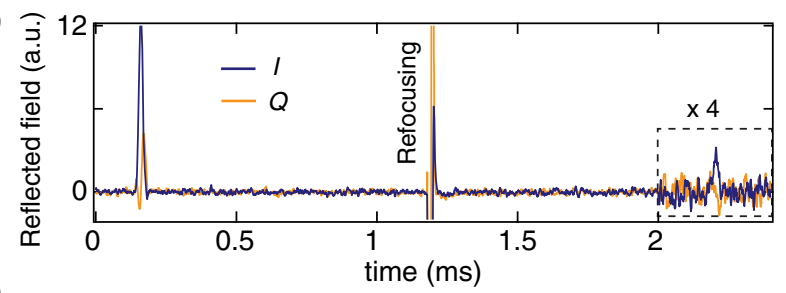

(b)
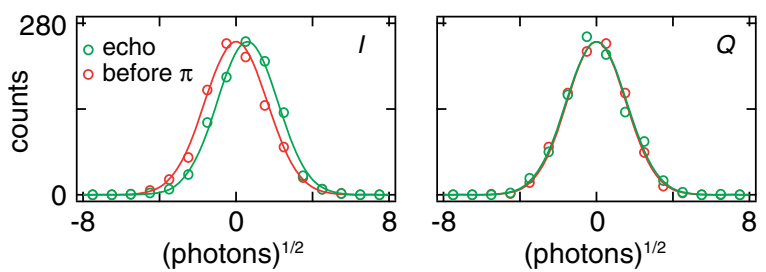

FIG. 4. Noise statistics of retrieved echo. (a) Average of $10^{3}$ single shot traces of reflected signals acquired with a repetition rate of $16 \mathrm{~s}$. The input and retrieved field contain 240 and 0.25 photons, respectively. In this run, the internal losses were slightly larger than in Fig. 3(b), leading to a lower value of $\zeta$ and a lower echo amplitude. (b) Histograms of signal quadratures before the refocusing $\pi$ pulse and on the echo. Each histogram sample is acquired by integrating signals for $100 \mu \mathrm{s}$ weighted by a normalized gaussian mode shape describing the echo. Solid curves are calculated Gaussians of the same area. 
A fully operational quantum memory requires increasing the ensemble cooperativity by a factor $\sim 30$, up to $C=1$. We argue that this can be achieved by simple design adjustments and without compromising $T_{2}^{E}$. First, $\kappa_{i}^{-1}$ and hence $C$ can be improved by a factor $5-10$ by using magnetic-field resilient superconductors [60,61]. Moreover, it is possible to increase the number of spins at fixed concentration by using a deeper implantation profile, up to $1 \mu \mathrm{m}$ as already shown [62], thus increasing $C$ by another factor $\sim 5$. A full quantum memory demonstration could then be achieved using the two-pulse protocol proposed in Refs. [36,37], and applied to store quantum states originating from a transmon-based quantum processor.

In conclusion, we have demonstrated the absorption of trains of weak microwave pulses consisting of only a few photons in a hybrid quantum device, their storage for $100 \mathrm{~ms}$, and their phase-coherent reemission without added noise. Our results illustrate the utility of clock transitions for efficient quantum memories with long storage times as well as memory reset via the Purcell effect.

We thank P. Seńat, D. Duet, and J.-C. Tack for the technical support, and are grateful for fruitful discussions within the Quantronics group. We acknowledge IARPA and Lincoln Labs for providing a JTWPA used in the measurements. We acknowledge support from the Horizon 2020 research and innovation program through Grant Agreement No. 771493 (LOQO-MOTIONS), and of the European Union through the Marie 365 Sklodowska Curie Grant Agreement No. 765267 (QuSCO), and of the Agence Nationale de la Recherche (ANR) through projects QIPSE, MIRESPIN (ANR 19 CE47 0011) and the Chaire Industrielle NASNIQ under contract ANR-17CHIN-0001 cofunded by Atos, and of the UK's Engineering and Physical Sciences Research Council through a Doctoral Training Award. T. S. was supported by the U.S. Department of Energy under Contract No. DE-AC02-05CH11231.

*patrice.bertet@cea.fr

[1] A. I. Lvovsky, B. C. Sanders, and W. Tittel, Nat. Photonics 3, 706 (2009).

[2] M. P. Hedges, J. J. Longdell, Y. Li, and M. J. Sellars, Nature (London) 465, 1052 (2010).

[3] M. Businger, A. Tiranov, K. T. Kaczmarek, S. Welinski, Z. Zhang, A. Ferrier, P. Goldner, and M. Afzelius, Phys. Rev. Lett. 124, 053606 (2020).

[4] P. Rabl, D. DeMille, J. M. Doyle, M. D. Lukin, R. J. Schoelkopf, and P. Zoller, Phys. Rev. Lett. 97, 033003 (2006).

[5] K. Tordrup, A. Negretti, and K. Mølmer, Phys. Rev. Lett. 101, 040501 (2008).

[6] C. Rigetti, J. M. Gambetta, S. Poletto, B. L. T. Plourde, J. M. Chow, A. D. Corcoles, J. A. Smolin, S. T. Merkel, J. R. Rozen, G. A. Keefe, M. B. Rothwell, M. B. Ketchen, and M. Steffen, Phys. Rev. B 86, 100506(R) (2012).
[7] R. Barends, J. Kelly, A. Megrant, D. Sank, E. Jeffrey, Y. Chen, Y. Yin, B. Chiaro, J. Mutus, C. Neill, P. O Malley, P. Roushan, J. Wenner, T. C. White, A. N. Cleland, and J. M. Martinis, Phys. Rev. Lett. 111, 080502 (2013).

[8] F. Arute et al., Nature (London) 574, 505 (2019).

[9] M. Reagor, W. Pfaff, C. Axline, R. W. Heeres, N. Ofek, K. Sliwa, E. Holland, C. Wang, J. Blumoff, K. Chou, M. J. Hatridge, L. Frunzio, M. H. Devoret, L. Jiang, and R. J. Schoelkopf, Phys. Rev. B 94, 014506 (2016).

[10] R. K. Naik, N. Leung, S. Chakram, P. Groszkowski, Y. Lu, N. Earnest, D. C. McKay, J. Koch, and D. I. Schuster, Nat. Commun. 8, 1904 (2017).

[11] T. A. Palomaki, J. W. Harlow, J. D. Teufel, R. W. Simmonds, and K. W. Lehnert, Nature (London) 495, 210 (2013).

[12] C. T. Hann, C.-L. Zou, Y. Zhang, Y. Chu, R. J. Schoelkopf, S. M. Girvin, and L. Jiang, Phys. Rev. Lett. 123, 250501 (2019).

[13] A. M. Tyryshkin, S. Tojo, J. J. L. Morton, H. Riemann, N. V. Abrosimov, P. Becker, H.-J. Pohl, T. Schenkel, M. L. W. Thewalt, K. M. Itoh, and S. A. Lyon, Nat. Mater. 11, 143 (2012).

[14] M. Steger, K. Saeedi, M. L. W. Thewalt, J. J. L. Morton, H. Riemann, N. V. Abrosimov, P. Becker, and H.-J. Pohl, Science 336, 1280 (2012).

[15] J. T. Muhonen, J. P. Dehollain, A. Laucht, F. E. Hudson, R. Kalra, T. Sekiguchi, K. M. Itoh, D. N. Jamieson, J. C. McCallum, A.S. Dzurak, and A. Morello, Nat. Nanotechnol. 9, 986 (2014).

[16] J. H. Wesenberg, A. Ardavan, G. A. D. Briggs, J. J. L. Morton, R. J. Schoelkopf, D. I. Schuster, and K. Mølmer, Phys. Rev. Lett. 103, 070502 (2009).

[17] A. Imamoglu, Phys. Rev. Lett. 102, 083602 (2009).

[18] J. J. L. Morton and P. Bertet, J. Magn. Reson. 287, 128 (2018).

[19] Y. Kubo, F. R. Ong, P. Bertet, D. Vion, V. Jacques, D. Zheng, A. Dreau, J.-F. Roch, A. Auffeves, F. Jelezko, J. Wrachtrup, M. F. Barthe, P. Bergonzo, and D. Esteve, Phys. Rev. Lett. 105, 140502 (2010).

[20] D. I. Schuster, A. P. Sears, E. Ginossar, L. DiCarlo, L. Frunzio, J. J. L. Morton, H. Wu, G. A. D. Briggs, B. B. Buckley, D. D. Awschalom, and R. J. Schoelkopf, Phys. Rev. Lett. 105, 140501 (2010).

[21] X. Zhu, S. Saito, A. Kemp, K. Kakuyanagi, S.-i. Karimoto, H. Nakano, W. J. Munro, Y. Tokura, M. S. Everitt, K. Nemoto, M. Kasu, N. Mizuochi, and K. Semba, Nature (London) 478, 221 (2011).

[22] Y. Kubo, C. Grezes, A. Dewes, T. Umeda, J. Isoya, H. Sumiya, N. Morishita, H. Abe, S. Onoda, T. Ohshima, V. Jacques, A. Dreau, J.-F. Roch, I. Diniz, A. Auffeves, D. Vion, D. Esteve, and P. Bertet, Phys. Rev. Lett. 107, 220501 (2011).

[23] R. Amsuss, C. Koller, T. Nbauer, S. Putz, S. Rotter, K. Sandner, S. Schneider, M. Schrambck, G. Steinhauser, H. Ritsch, J. Schmiedmayer, and J. Majer, Phys. Rev. Lett. 107, 060502 (2011).

[24] E. Abe, H. Wu, A. Ardavan, and J. J. L. Morton, Appl. Phys. Lett. 98, 251108 (2011).

[25] V. Ranjan, G. de Lange, R. Schutjens, T. Debelhoir, J. P. Groen, D. Szombati, D. J. Thoen, T. M. Klapwijk, R. Hanson, and L. DiCarlo, Phys. Rev. Lett. 110, 067004 (2013). 
[26] S. Probst, H. Rotzinger, S. Wunsch, P. Jung, M. Jerger, M. Siegel, A. V. Ustinov, and P. A. Bushev, Phys. Rev. Lett. 110, 157001 (2013).

[27] H. Huebl, C. W. Zollitsch, J. Lotze, F. Hocke, M. Greifenstein, A. Marx, R. Gross, and S. T. B. Goennenwein, Phys. Rev. Lett. 111, 127003 (2013).

[28] A. J. Sigillito, H. Malissa, A. M. Tyryshkin, H. Riemann, N. V. Abrosimov, P. Becker, H.-J. Pohl, M. L. W. Thewalt, K. M. Itoh, J. J. L. Morton, A. A. Houck, D. I. Schuster, and S. A. Lyon, Appl. Phys. Lett. 104, 222407 (2014).

[29] Y. Tabuchi, S. Ishino, T. Ishikawa, R. Yamazaki, K. Usami, and Y. Nakamura, Phys. Rev. Lett. 113, 083603 (2014).

[30] Y.-H. Chen, X. Fernandez-Gonzalvo, and J. J. Longdell, Phys. Rev. B 94, 075117 (2016).

[31] B. C. Rose, A. M. Tyryshkin, H. Riemann, N. V. Abrosimov, P. Becker, H.-J. Pohl, M. L.W. Thewalt, K. M. Itoh, and S. A. Lyon, Phys. Rev. X 7, 031002 (2017).

[32] J. R. Ball, Y. Yamashiro, H. Sumiya, S. Onoda, T. Ohshima, J. Isoya, D. Konstantinov, and Y. Kubo, Appl. Phys. Lett. 112, 204102 (2018).

[33] E. M. Purcell, Phys. Rev. 69, 681 (1946).

[34] A. Bienfait, J. Pla, Y. Kubo, X. Zhou, M. Stern, C.-C. Lo, C. Weis, T. Schenkel, D. Vion, D. Esteve, J. Morton, and P. Bertet, Nature (London) 531, 74 (2016).

[35] C. Eichler, A. J. Sigillito, S. A. Lyon, and J. R. Petta, Phys. Rev. Lett. 118, 037701 (2017).

[36] M. Afzelius, N. Sangouard, G. Johansson, M. U. Staudt, and C. M. Wilson, New J. Phys. 15, 065008 (2013).

[37] B. Julsgaard, C. Grezes, P. Bertet, and K. Mølmer, Phys. Rev. Lett. 110, 250503 (2013).

[38] See Supplemental Material at http://link.aps.org/ supplemental/10.1103/PhysRevLett.125.210505 for additional details on setup, experiments, and theory.

[39] J. Ruggiero, J.-L. Le Gouet, C. Simon, and T. Chaneliere, Phys. Rev. A 79, 053851 (2009).

[40] V. Damon, M. Bonarota, A. Louchet-Chauvet, T. Chaneliere, and J.-L. Le Gouet, New J. Phys. 13, 093031 (2011).

[41] H. Wu, R. E. George, J. H. Wesenberg, K. Mølmer, D. I. Schuster, R. J. Schoelkopf, K. M. Itoh, A. Ardavan, J. J. L. Morton, and G. A. D. Briggs, Phys. Rev. Lett. 105, 140503 (2010).

[42] C. Grezes, B. Julsgaard, Y. Kubo, M. Stern, T. Umeda, J. Isoya, H. Sumiya, H. Abe, S. Onoda, T. Ohshima, V. Jacques, J. Esteve, D. Vion, D. Esteve, K. Mølmer, and P. Bertet, Phys. Rev. X 4, 021049 (2014).

[43] S. Probst, H. Rotzinger, A. V. Ustinov, and P. A. Bushev, Phys. Rev. B 92, 014421 (2015).

[44] C. Grezes, B. Julsgaard, Y. Kubo, W. L. Ma, M. Stern, A. Bienfait, K. Nakamura, J. Isoya, S. Onoda, T. Ohshima, V. Jacques, D. Vion, D. Esteve, R. B. Liu, K. Mølmer, and P. Bertet, Phys. Rev. A 92, 020301(R) (2015).

[45] C. Grezes, Y. Kubo, B. Julsgaard, T. Umeda, J. Isoya, H. Sumiya, H. Abe, S. Onoda, T. Ohshima, K. Nakamura,
I. Diniz, A. Auffeves, V. Jacques, J.-F. Roch, D. Vion, D. Esteve, K. Mølmer, and P. Bertet, C. R. Phys. Quantum Microwaves/Micro-ondes Quantiques 17, 693 (2016).

[46] M. H. Mohammady, G. W. Morley, and T. S. Monteiro, Phys. Rev. Lett. 105, 067602 (2010).

[47] R. E. George, W. Witzel, H. Riemann, N. V. Abrosimov, N. Notzel, M. L. W. Thewalt, and J. J. L. Morton, Phys. Rev. Lett. 105, 067601 (2010).

[48] G. Wolfowicz, A. M. Tyryshkin, R. E. George, H. Riemann, N. V. Abrosimov, P. Becker, H.-J. Pohl, M. L. W. Thewalt, S. a. Lyon, and J. J. L. Morton, Nat. Nanotechnol. 8, 561 (2013).

[49] P. A. Mortemousque, S. Berger, T. Sekiguchi, C. Culan, R. G. Elliman, and K. M. Itoh, Phys. Rev. B 89, 155202 (2014).

[50] M. Zhong, M. P. Hedges, R. L. Ahlefeldt, J. G. Bartholomew, S. E. Beavan, S. M. Wittig, J. J. Longdell, and M. J. Sellars, Nature (London) 517, 177 (2015).

[51] A. Ortu, A. Tiranov, S. Welinski, F. Frwis, N. Gisin, A. Ferrier, P. Goldner, and M. Afzelius, Nat. Mater. 17, 671 (2018).

[52] M. Shiddiq, D. Komijani, Y. Duan, A. Gaita-Ario, E. Coronado, and S. Hill, Nature (London) 531, 348 (2016).

[53] C. D. Weis, C. C. Lo, V. Lang, A. M. Tyryshkin, R. E. George, K. M. Yu, J. Bokor, S. A. Lyon, J. J. L. Morton, and T. Schenkel, Appl. Phys. Lett. 100, 172104 (2012).

[54] A. Bienfait, J. J. Pla, Y. Kubo, M. Stern, X. Zhou, C. C. Lo, C. D. Weis, T. Schenkel, M. L. W. Thewalt, D. Vion, D. Esteve, B. Julsgaard, K. Mølmer, J. J. L. Morton, and P. Bertet, Nat. Nanotechnol. 11, 253 (2016).

[55] C. Macklin, K. OBrien, D. Hover, M. E. Schwartz, V. Bolkhovsky, X. Zhang, W. D. Oliver, and I. Siddiqi, Science 350, 307 (2015).

[56] J. J. Pla, A. Bienfait, G. Pica, J. Mansir, F. A. Mohiyaddin, Z. Zeng, Y. M. Niquet, A. Morello, T. Schenkel, J. J. L. Morton, and P. Bertet, Phys. Rev. Applied 9, 044014 (2018).

[57] J. Mansir, P. Conti, Z. Zeng, J. J. Pla, P. Bertet, M. W. Swift, C. G. Van de Walle, M. L. W. Thewalt, B. Sklenard, Y. M. Niquet, and J. J. L. Morton, Phys. Rev. Lett. 120, 167701 (2018).

[58] C. W. Thiel, W. R. Babbitt, and R. L. Cone, Phys. Rev. B 85, 174302 (2012).

[59] V. Ranjan et al. (to be published).

[60] S. E. d. Graaf, A. V. Danilov, A. Adamyan, T. Bauch, and S. E. Kubatkin, J. Appl. Phys. 112, 123905 (2012).

[61] N. Samkharadze, A. Bruno, P. Scarlino, G. Zheng, D. P. DiVincenzo, L. DiCarlo, and L. M. K. Vandersypen, Phys. Rev. Applied 5, 044004 (2016).

[62] B. Albanese, S. Probst, V. Ranjan, C. W. Zollitsch, M. Pechal, A. Wallraff, J. J. L. Morton, D. Vion, D. Esteve, E. Flurin, and P. Bertet, Nat. Phys. 16, 751 (2020). 